\title{
Uma análise do impacto da utilização de técnicas de gamificação como estratégia didática no aprendizado dos alunos
}

\author{
Amaury Nogueira Neto ${ }^{1}$, Alan Pedro da Silva1 ${ }^{1}$, Ig Ibert Bittencourt ${ }^{1}$ \\ ${ }^{1}$ Instituto de Computação - Universidade Federal de Alagoas (UFAL) \\ CEP 57072-970 Maceió - AL - Brasil \\ \{ann, alanpedro, ig.ibert \}@ic.ufal.br
}

\begin{abstract}
As a result there are many studies that claim that the use of gamification provides many advantages in the field of education, but very few, so far, we empirically prove that such benefits are due only the use of gamification techniques, an experimental research was conducted in order to analyze the first year high school students with the intention to assess what is the impact of using gamification in relation to their learning from the point of view of educators and researchers in the mathematical discipline of the teaching context for the first year of high school in public schools in Brazil. The results point to an improvement in the performance of groups of students analyzed, making it possible to conclude that the gamification as a teaching strategy contributes positively on student achievement.
\end{abstract}

Resumo. Em decorrência de haverem muitos trabalhos que afirmam que o uso de gamificação possibilita muitas vantagens no campo da educação, porém muito poucos, até o momento, que comprovem empiricamente que tais vantagens são decorrentes apenas do uso de técnicas de gamificação, foi realizada uma pesquisa experimental a fim de analisar alunos do primeiro ano do ensino médio com a intenção de avaliar qual é o impacto do uso de gamificação em relação ao seu aprendizado, do ponto de vista de educadores e pesquisadores, no contexto do ensino da disciplina de matemática para o primeiro ano do ensino médio em escolas públicas do Brasil. Os resultados obtidos apontam para uma melhora no rendimento dos grupos de alunos analisados, o que possibilitou concluir que o uso de gamificação como estratégia didática contribui positivamente no rendimento dos alunos.

\section{Introdução}

Segundo Kapp (2012), o termo gamificação tem por significado o uso de mecânicas, estéticas e pensamentos de jogos a fim de promover o engajamento de pessoas, motivação para a ação, contribuindo para a aprendizagem e resolução de problemas em diferentes contextos de aplicação, tais como comércio, aplicativos eletrônicos e educação. $\mathrm{O}$ autor destaca ainda que o interesse pelo tema tem crescido consideravelmente nos últimos anos, atribuindo a este fato o potencial da gamificação para influenciar, engajar e motivar pessoas.

Ainda segundo o autor, mesmo que o termo gamificação tenha sido usado apenas recentemente, não é um conceito novo - na educação, por exemplo, podemos lembrar de um típico caso de uso de gamificação que muitos de nós até já vivenciamos 
quando crianças, quando éramos recompensados com objetos como estrelas por bom desempenho ou bom comportamento. Porém, ainda que os conceitos não possam ser considerados como novos, pode-se dizer que os estudos acerca destes conceitos, quando comparados com estudos de outras ciências mais tradicionais, são relativamente recentes. Percebe-se que o uso de dinâmicas de jogos em ambientes que não são jogos tem sido cada vez mais frequente nos mais diversos campos, a exemplo dos já citados anteriormente, possibilitando uma melhora no seu rendimento, conforme aponta Kapp (2012).

Em um mapeamento sistemático realizado por Borges et al (2013) acerca da gamificação na educação, foi possível identificar sete objetivos principais para o uso de gamificação que foram enfatizados pelos autores, a saber o aprimoramento de habilidades; proposta de desafios para dar propósito/contexto para a aprendizagem; engajamento de alunos em atividades mais participativas, interativas e interessantes; maximização do aprendizado determinado conteúdo; promoção da mudança de comportamento através da recompensa por ações adequadas e penalização das inadequadas; oferecimento de mecanismos de socialização e aprendizagem em grupo e discussões acerca dos benefícios de gamificação na motivação dos alunos para propor soluções aos diversos problemas de aprendizagem.

Segundo os autores, a grande maioria dos artigos selecionados no mapeamento sistemático (92\% do total) cita a promoção do engajamento dos estudantes como justificativa para o uso de tais técnicas. Ainda segundo os autores, a gamificação tem como pilares fundamentais pontos, quadros de líderes e troféus, podendo ser utilizada, por exemplo, para aprofundar o conhecimento sobre um determinado produto e/ou facilitar e estimular a aprendizagem. Uma vez que esta técnica vem sendo utilizada com relativo sucesso em diversas áreas, percebe-se a necessidade de avaliar o quanto ela pode ser considerada positiva quando utilizada como estratégia didática, havendo a necessidade de avaliar qual o impacto do uso desta técnica na aprendizagem do aluno.

Como trabalhos relacionados, podemos citar Jucá e Rolim (2014), que relatam que o uso de gamificação na educação contribui de maneira significativa na motivação dos alunos na disciplina de empreendedorismo, e Pereira e Pimentel (2014), que propõem um laboratório virtual gamificado para a prática experimental no ensino de química. Compreende-se que o presente trabalho é relevante pelo fato de haverem muitos trabalhos que afirmam que o uso de gamificação possibilita muitas vantagens no campo da educação, mas poucos que comprovem, empiricamente, que tais vantagens são decorrentes apenas do uso de técnicas de gamificação.

Partindo-se desta perspectiva, observou-se a possibilidade contribuição da informática no campo da educação, com o intuito de analisar viabilidade do uso de estratégias de ensino diferentes das estratégias tradicionais, baseadas no uso de gamificação para o ensino. Buscou-se avaliar o impacto da contribuição da informática na educação através do uso técnicas de gamificação em um ambiente computacional. A fim de se encontrar um cenário para a realização da presente pesquisa, buscou-se inicialmente compreender a atual situação da educação no Brasil através da análise de seus indicadores educacionais.

A fim de se ter uma ideia na real situação da educação no Brasil, foi utilizado como parâmetro a análise do o IDEB - Índice de Desenvolvimento da Educação Básica. O IDEB é um indicador criado pelo Governo Federal do Brasil com o intuito de medir a 
qualidade do ensino nas escolas públicas, sendo calculado com base no aprendizado dos alunos nas disciplinas de português e matemática e na taxa de aprovação escolar, conforme aponta INEP (2015). O último IDEB foi realizado no ano de 2013, e de acordo com índice, o ensino médio da rede pública brasileira não atingiu a meta, que era de 3.6 pontos, estando atualmente em 3.4 e não tendo alcançado 6.0 pontos (que é a média correspondente ao sistema educacional dos países desenvolvidos e objetivo único até o ano de 2022), o que demonstra que o país precisa melhorar a sua situação para garantir mais alunos aprendendo e com um fluxo escolar adequado. No estado de Alagoas, a realidade é ainda mais preocupante - mesmo o estado tendo melhorado seu IDEB de 2.6 para 2.8, ainda está abaixo da média nacional.

Tendo-se em vista a realidade da educação no Brasil, e levando-se em consideração que o IDEB está diretamente relacionado com o aprendizado das disciplinas de português e matemática, optou-se por avaliar qual o impacto do uso de técnicas de gamificação no ensino de matemática para alunos do primeiro ano do ensino médio de escolas públicas. Procurou-se identificar se o uso de gamificação em um ambiente de educação computacional afeta o aprendizado de matemática de alunos no contexto do primeiro ano do ensino médio em escolas públicas, com vistas a aferir se este fator viabilizaria melhoras no rendimento dos alunos na referida disciplina.

\section{Contextualização}

Acerca da avaliação do conhecimento escolar assimilado pelos alunos, a Lei de Diretrizes e Bases da Educação - LDB (Brasil, 1998), determina que sejam observados critérios contínuos e cumulativos na atuação do educando, priorizando aspectos qualitativos aos quantitativos, e de resultados ao longo de um período sobre os de eventuais avaliações finais.

Tomando como métrica base a avaliação contínua e cumulativa do aprendizado, amplamente utilizada para a verificação da assimilação do conhecimento escolar de educandos, conforme determinações da LDB, pode-se associar o bom desempenho dos alunos à correta assimilação dos conteúdos.

Sendo assim, a principal questão de pesquisa do presente projeto tem por objetivo determinar e comparar a eficácia do uso de técnicas de gamificação para o aprendizado da disciplina de matemática para alunos do primeiro ano do ensino médio de escolas públicas, através de avaliações contínuas e cumulativas da aprendizagem dos alunos, a fim de responder à seguinte questão: $\mathrm{O}$ uso de técnicas de gamificação, para 0 ensino da disciplina de matemática para alunos do primeiro ano do ensino médio de escolas públicas, influencia no aprendizado dos alunos na disciplina? O que nos leva às seguintes hipóteses:

- H1-0: O uso de técnicas de gamificação para o ensino da disciplina de matemática para alunos do primeiro ano do ensino médio de escolas públicas não influencia no rendimento dos alunos.

- H1-1: O uso de técnicas de gamificação para o ensino da disciplina de matemática para alunos do primeiro ano do ensino médio de escolas públicas influencia no rendimento dos alunos.

Caso a hipótese nula seja refutada - indicando que o uso de gamificação como estratégia didática influencia no rendimento dos alunos - os resultados do experimento 
serão analisados com vistas a aferir qual é o impacto do uso da gamificação no aprendizado no contexto deste experimento.

Formalmente, as duas hipóteses descritas anteriormente podem ser definidas conforme a Tabela 1, onde R representa a média de rendimento do grupo de alunos, Gd representa Gamificação Desativada e Ga, Gamificação Ativada.

Tabela 1. Definição formal das hipóteses

\begin{tabular}{|c|c|c|}
\hline Hipótese & Hipótese Nula & Hipótese Alternativa \\
\hline $\mathrm{H} 1$ & $\mathrm{H} 1.0: \mathrm{R}(\mathrm{Gd})=\mathrm{R}(\mathrm{Ga})$ & $\mathrm{H} 1.1: \mathrm{R}(\mathrm{Gd}) \neq \mathrm{R}(\mathrm{Ga})$ \\
\hline
\end{tabular}

Com o objetivo de aferir o impacto do uso de gamificação na educação, foi realizada uma pesquisa experimental, a fim de analisar alunos do primeiro ano do ensino médio com a intenção de avaliar qual é o impacto do uso de gamificação em relação ao seu aprendizado, mais especificamente com respeito ao seu rendimento, do ponto de vista de educadores e pesquisadores, no contexto do ensino da disciplina de matemática para o primeiro ano do ensino médio. Para tanto, foram comparados os resultados de avaliações periódicas do aprendizado em grupos de alunos onde foram utilizadas técnicas de gamificação como estratégias didáticas com grupos de alunos onde não foram utilizadas tais estratégias.

A partir da verificação dos resultados do experimento, foi possível elencar algumas conclusões acerca do impacto da utilização de técnicas de gamificação como estratégia didática no aprendizado dos grupos de alunos. A execução do experimento envolveu os seguintes passos:

- Estudo dos assuntos por parte dos alunos;

- Coleta dos dados (através de avaliações); e

- Análise estatística dos resultados.

Seguindo com o planejamento, foram definidas as variáveis contidas no experimento. Como variáveis independentes, foram elencadas:

- Utilização de recursos de gamificação: que pode ser ativada ou desativada.

- Assunto: diz respeito ao conteúdo a ser visto pelo aluno.

Os níveis dos fatores estão definidos de acordo com a Tabela 2: 
CBIE-LACLO 2015

Anais do XXVI Simpósio Brasileiro de Informática na Educação (SBIE 2015)

\begin{tabular}{|l|c|}
\hline \multicolumn{1}{|c|}{ Fator } & Níveis \\
\hline Utilização de recursos de gamificação & $\begin{array}{c}\text { Desativado } \\
\text { Ativado }\end{array}$ \\
\hline Assunto & $\begin{array}{c}\text { Conjuntos e funções } \\
\text { Função polinomial do primeiro grau }\end{array}$ \\
\hline
\end{tabular}

Como possíveis variáveis independentes, foram elencadas:

- Tempo de estudo (foi estipulado um tempo padrão de $2 \mathrm{~h}$ semanais para cada aluno)

- Foram padronizados todos os aspectos relacionados à conectividade, configuração dos computadores e ambiente de estudos.

Como variáveis resposta, foram elencados:

- Rendimento dos alunos: no decorrer do experimento, serão realizadas avaliações objetivas do aprendizado do aluno, através da aplicação de testes periódicos com vistas a acompanhar o progresso de seu aprendizado.

Como unidades experimentais, temos 60 alunos dos primeiro anos do ensino médio do Instituto Federal de Alagoas, campus Satuba no ano letivo de 2015. No tocante à metodologia, foram seguidas as recomendações de Montgomery (2001) e Juristo e Moreno (2001).

\section{Design do Experimento}

Levando em consideração as diversas classificações de experimento (JURISTO e MORENO, 2001), o presente experimento é classificado como fatorial completo com repetições. O experimento é repetido 120 vezes. Dessa forma, como o experimento possui dois fatores, que são o uso de Gamificação, com 2 níveis (ativado e desativado) e Assunto, com 2 níveis (conjuntos e funções e função polinomial do primeiro grau), multiplicando esses níveis resulta em um total de 4 tratamentos, sendo executado 30 vezes, totalizando 120 execuções, conforme ilustrado na tabela 3.

Tabela 3. Definição dos ensaios

\begin{tabular}{|c|l|l|}
\hline $\mathbf{N}^{\circ}$ do Tratamento & Gamificação & Assunto \\
\hline 1 & Ativada & Função polinomial do primeiro grau \\
\hline 2 & Desativada & Função polinomial do primeiro grau \\
\hline 3 & Ativada & Conjuntos e funções \\
\hline 4 & Desativada & Conjuntos e funções \\
\hline
\end{tabular}

Em cada replicação do experimento, os dados de entrada serão filtrados a partir de avaliações do aprendizado dos alunos. Como instrumentação, tem-se a plataforma Meu Tutor (2015) - plataforma educacional gamificada adaptativa focada na qualidade do ensino e desempenho dos alunos. 


\section{Ameaças à validade}

Quanto às ameaças à validade do experimento, não foram identificadas ameaças à validade interna ou do constructo, porém, tem-se que as unidades experimentais da pesquisa foram selecionadas a partir de uma única fonte (uma única escola), que pode ter características próprias que não valem para todas as demais escolas do país. Logo, há uma ameaça à validade externa na interação de seleção e tratamento, o que dificulta a generalização dos resultados além do escopo estudado.

Adicionalmente, temos que os dados serão retirados a partir de uma janela temporal, o que pode significar que apenas uma determinada amostra da população de alunos será utilizada. Dados específicos desta janela temporal escolhida podem influenciar nos resultados, o que configura uma ameaça à validade externa na interação de história e tratamento. Por exemplo: a janela temporal pode englobar apenas assuntos de um bimestre do primeiro ano do ensino médio.

O uso de gamificação atrelada a uma plataforma educacional online pode alterar o nível de contato do aluno recursos de gamificação, pois passam a figurar no experimento fatores que podem influenciar diretamente nos resultados, como por exemplo, os níveis de conhecimento do aluno sobre informática e sua afinidade com recursos tecnológicos. Além disto, com uma amostra muito pequena, a significância estatística do experimento pode não ser suficiente, gerando uma ameaça à validade de conclusão.

\section{Resultados}

\subsection{Apresentação dos dados}

Para garantir a privacidade e confidencialidade dos alunos avaliados, foram retirados quaisquer dados que pudessem identificar os sujeitos participantes da pesquisa. A tabela 4 mostra o rendimento dos alunos do grupo 1 , e a tabela 5, do grupo 2, sendo cada grupo composto por 30 alunos, totalizando 60 alunos participantes. A fim de aferir o rendimento, ao final de cada assunto, foram aplicadas avaliações escritas para cada aluno.

Tabela 4. Rendimento dos alunos do grupo 1

\begin{tabular}{|c|c|c|}
\hline Grupo 1 & $\begin{array}{c}\text { Assunto: Conjuntos e funções (com } \\
\text { gamificação ativada) }\end{array}$ & $\begin{array}{c}\text { Assunto: Função polinomial do primeiro grau (sem } \\
\text { gamificação ativada) }\end{array}$ \\
\hline Aluno 01 & 3 & 4,66 \\
\hline Aluno 02 & 4 & 2,66 \\
\hline Aluno 03 & 8,5 & 0,00 \\
\hline Aluno 04 & 7,5 & 1,00 \\
\hline Aluno 05 & 3,5 & 1,33 \\
\hline Aluno 06 & 8,5 & 9,00 \\
\hline Aluno 07 & 5 & 0,00 \\
\hline Aluno 08 & 2 & 0,00 \\
\hline Aluno 09 & 6 & 4,66 \\
\hline Aluno 10 & 4 & 2,60 \\
\hline Aluno 11 & 6 & 2,60 \\
\hline
\end{tabular}


CBIE-LACLO 2015

Anais do XXVI Simpósio Brasileiro de Informática na Educação (SBIE 2015)

\begin{tabular}{|l|c|c|} 
Aluno 12 & 6 & 6,00 \\
\hline Aluno 13 & 3,5 & 0,00 \\
\hline Aluno 14 & 5,5 & 2,60 \\
\hline Aluno 15 & 5 & 0,00 \\
\hline Aluno 16 & 3 & 4,66 \\
\hline Aluno 17 & 4,5 & 3,33 \\
\hline Aluno 18 & 9,5 & 10,00 \\
\hline Aluno 19 & 7,5 & 1,00 \\
\hline Aluno 20 & 3,5 & 1,33 \\
\hline Aluno 21 & 8,5 & 9,00 \\
\hline Aluno 22 & 5 & 0,00 \\
\hline Aluno 23 & 2 & 0,00 \\
\hline Aluno 24 & 6 & 4,66 \\
\hline Aluno 25 & 4 & 2,60 \\
\hline Aluno 26 & 6 & 2,60 \\
\hline Aluno 27 & 6 & 6,00 \\
\hline Aluno 28 & 3,5 & 0,00 \\
\hline Aluno 29 & 5,5 & 2,60 \\
\hline Aluno 30 & 5 & 0,00 \\
\hline
\end{tabular}

Tabela 5. Rendimento dos alunos do grupo 2

\begin{tabular}{|l|c|c|}
\hline Grupo 2 & $\begin{array}{c}\text { Assunto: Conjuntos e funções (sem } \\
\text { gamificação ativada) }\end{array}$ & $\begin{array}{c}\text { Assunto: Função polinomial do primeiro grau (com } \\
\text { gamificação ativada) }\end{array}$ \\
\hline Aluno 01 & 8 & 10,00 \\
\hline Aluno 02 & 9 & 10,00 \\
\hline Aluno 03 & 5 & 8,00 \\
\hline Aluno 04 & 1,5 & 10,00 \\
\hline Aluno 05 & 4,5 & 10,00 \\
\hline Aluno 06 & 5,5 & 9,32 \\
\hline Aluno 07 & 4 & 9,32 \\
\hline Aluno 08 & 3,5 & 8,00 \\
\hline Aluno 09 & 7,5 & 9,32 \\
\hline Aluno 10 & 3 & 10,00 \\
\hline Aluno 11 & 3,5 & 9,32 \\
\hline Aluno 12 & 8 & 10,00 \\
\hline Aluno 13 & 9 & 10,00 \\
\hline Aluno 14 & 5 & 8,00 \\
\hline Aluno 15 & 1,5 & 10,00 \\
\hline Aluno 16 & 4,5 & 10,00 \\
\hline Aluno 17 & 5,5 & 9,32 \\
\hline Aluno 18 & 4,5 & 9,32 \\
\hline Aluno 19 & & 8,00 \\
\hline Aluno 20 & 5 & 9,32 \\
\hline
\end{tabular}


CBIE-LACLO 2015

Anais do XXVI Simpósio Brasileiro de Informática na Educação (SBIE 2015)

\begin{tabular}{|l|c|c|} 
Aluno 21 & 3 & 10,00 \\
\hline Aluno 22 & 3,5 & 9,32 \\
\hline Aluno 23 & 8 & 10,00 \\
\hline Aluno 24 & 9 & 10,00 \\
\hline Aluno 25 & 5 & 8,00 \\
\hline Aluno 26 & 1,5 & 10,00 \\
\hline Aluno 27 & 4,5 & 10,00 \\
\hline Aluno 28 & 5,5 & 9,32 \\
\hline Aluno 29 & 4 & 9,32 \\
\hline Aluno 30 & 3,5 & 8,00 \\
\hline
\end{tabular}

Na tabela 6, é possível ver dados referentes às médias gerais dos grupos participantes do estudo.

Tabela 6. Médias gerais dos grupo participantes do estudo

\begin{tabular}{|l|c|c|}
\hline Assunto & Gamificação & Rendimento médio do grupo \\
\hline Função polinomial do primeiro grau & Desativada & 2,83 \\
\hline Funções e conjuntos & Desativada & 5,03 \\
\hline Função polinomial do primeiro grau & Ativada & 9,37 \\
\hline Funções e conjuntos & Ativada & 5,25 \\
\hline
\end{tabular}

\subsection{Análise dos resultados}

A fim de se obter uma melhor compreensão das informações obtidas, os dados foram analisados estatisticamente para a construção dos gráficos de Efeitos de Pareto, de Cubo e de Efeitos Principais, sendo tais gráficos ilustrados nas figuras 1, 2 e 3.

Figura 1. Gráfico de Pareto dos Efeitos

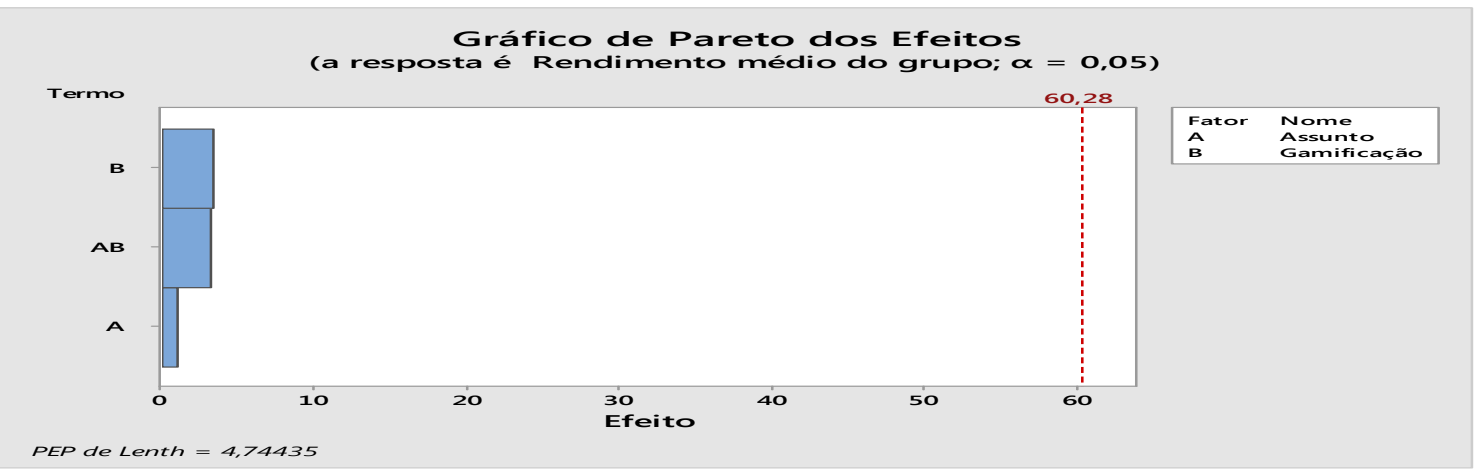

Figura 2. Gráfico de cubo para rendimento médio do grupo

Gráfico de Cubo (médias ajustadas) para Rendimento médio do grupo

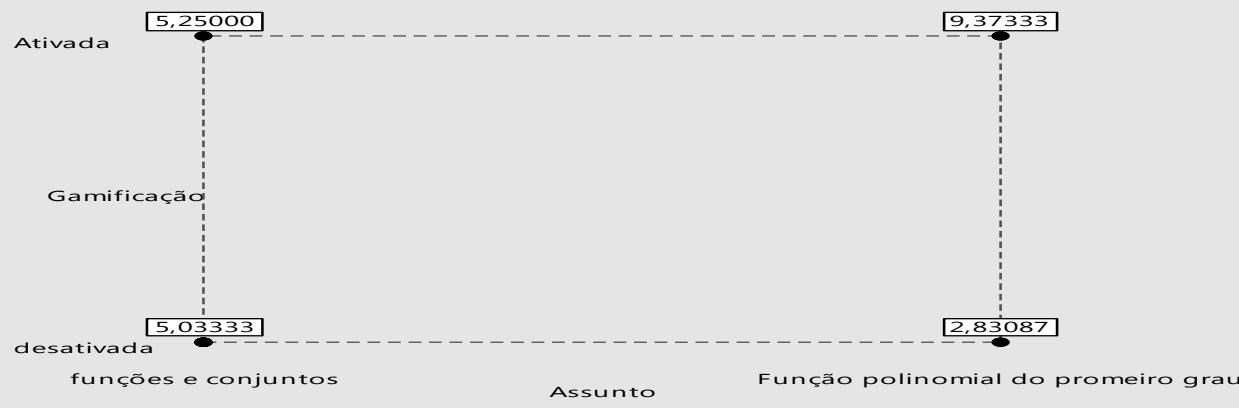


Figura 3. Gráfico de efeitos principais para rendimento médio do grupo

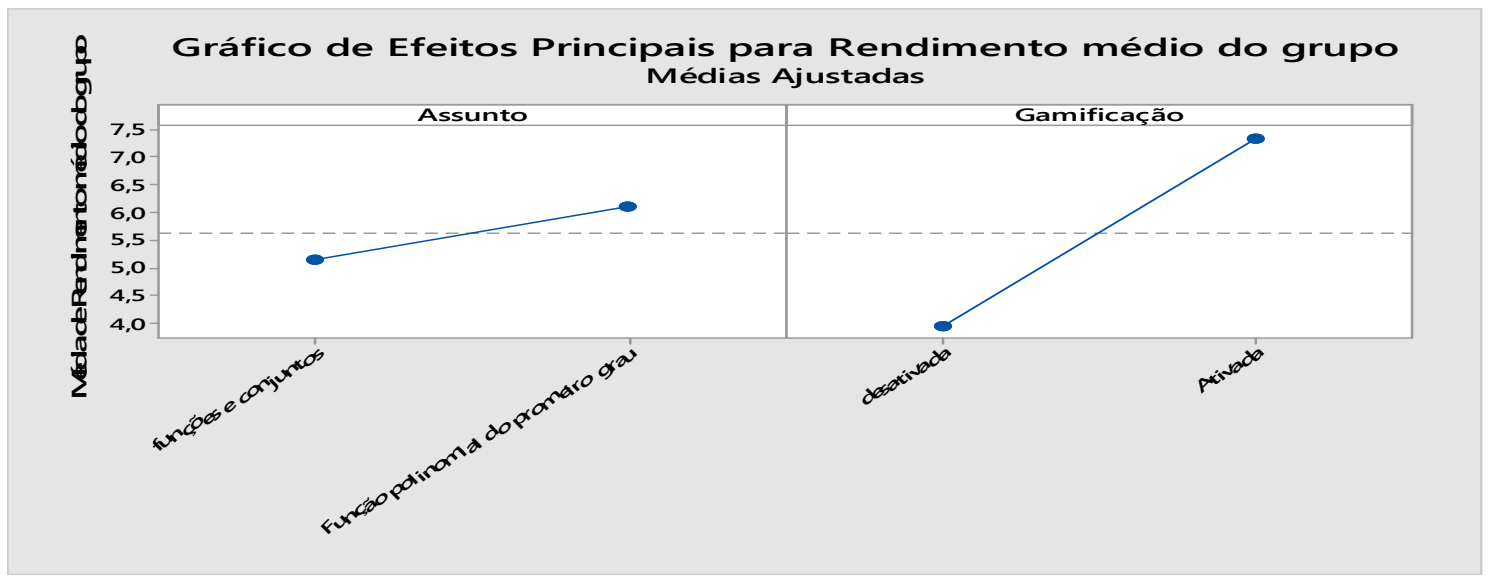

A partir da análise dos resultados, é possível perceber uma tendência de melhora no rendimento da variável resposta (rendimento dos alunos) à medida em que recursos de gamificação são ativados, e uma tendência oposta quando tais recursos não são utilizados. Tais informações serviram de subsídio para a seção seguinte, que é a discussão dos resultados obtidos. Em relação a outros trabalhos da área, considera-se que o presente trabalho tem uma contribuição significativa no sentido de prover subsídios estatísticos que corroborem para justificar o uso de gamificação como estratégia didática.

\subsection{Discussão dos resultados}

O presente trabalho teve como objetivo aferir se o uso de técnicas de gamificação para o ensino da disciplina de matemática para alunos do primeiro ano do ensino médio de escolas públicas influencia no aprendizado dos alunos na disciplina. Tal problemática levou às seguintes hipóteses:

- H1-0: O uso de técnicas de gamificação para o ensino da disciplina de matemática para alunos do primeiro ano do ensino médio de escolas públicas não influencia no rendimento dos alunos.

- H1-1: O uso de técnicas de gamificação para o ensino da disciplina de matemática para alunos do primeiro ano do ensino médio de escolas públicas influencia no rendimento dos alunos.

A partir da análise dos dados obtidos através da realização do experimento, notou-se uma melhora significativa no rendimento dos alunos quando usados recursos de gamificação como estratégia didática. Pode-se inferir, a partir dos dados obtidos, que o uso de gamificação, nos dois assuntos abordados, contribuiu para um melhor rendimento médio dos grupos de alunos, o que refuta a hipótese nula, indicando que o uso de gamificação como estratégia didática influencia no rendimento dos alunos. Uma vez que os resultados obtidos apontam para uma melhora no rendimento dos grupos de alunos analisados, é possível dizer que o uso de gamificação como estratégia didática contribui positivamente no rendimento dos alunos.

\section{Conclusão}

O presente experimento ainda encontra-se em execução e espera-se concluí-lo com 480 interações. Os dados obtidos até o momento tem apontado para a conclusão de que o 
uso de gamificação como estratégia didática pode contribuir de maneira positiva e significativa no sentido de melhorar o rendimento dos alunos na disciplina matemática no primeiro ano do ensino médio em escolas públicas. Como trabalhos futuros, podem ser sugeridas ampliações e diversificação do escopo das unidades experimentais utilizadas na pesquisa, uma vez que esta foi realizada em apenas uma escola e pode não retratar a totalidade da real situação da educação do país. O presente trabalho tem apontado no sentido de comprovar a efetividade do uso de técnicas de gamificação, e espera-se que trabalhos futuros possam enriquecer a comunidade no sentido de ampliar as possibilidades de tais benefícios.

\section{Referências}

BRASIL. Lei de Diretrizes e Bases da Educação: Lei nº 9.394/96 - 24 de dez. 1996. Estabelece as diretrizes e bases da educação nacional. Brasília, 1998.

BORGES, S. S., Reis, H. M., Durelli, V. H. S., Isotani, S. Gamificação Aplicada à Educação: Um Mapeamento Sistemático. In: Congresso Brasileiro de Informática na Educação, II, 2013, cidade, Anais do XXIV Simpósio Brasileiro de Informática na Educação, Campinas-SP: Sociedade Brasileira de Computação, 2013.

INEP. Indicadores educacionais e dados consolidados. Brasília, 2015.

JUCÁ, P., ROLIM, G. (2014). Aplicação da Gamificação na Disciplina de Empreendedorismo. In: XXI Workshop sobre Educação em Computação (WEI) do XXXIII Congresso da Sociedade Brasileira de Computação. Maceió. 2014

JURISTO, N.; MORENO, A. M. Basics of Software Engineering Experimentation. $1^{\mathrm{a}}$. ed. Dordrecht: Kluwer Academic Publishers, 2001.

KAPP, K. M. The Gamification of learning and instruction: Game-based methods and strategies for training and education. Pfeiffer. Hoboken, NJ. 2012.

MEU TUTOR. Disponível em <http://www.meututor.com.br>. Acesso em 19 abr 2015.

MONTGOMERY, Douglas C. Design and Analysis of Experiments, John Wiley \& Sons, Inc., New York. 2001.

PEREIRA, Saulo R. de C., PIMENTEL, Edson P.Laboratório Virtual Gamificado para a Prática Experimental no Ensino de Química. In: Latin American Conference on Learning Objects and Technologies - LACLO, Anais do 9th Latin American Conference on Learning Objects and Technologies, Manizales, Colombia, 2014. 